\title{
РАДИОСПЕКТРОСКОПИЯ
}

\author{
PACS numbers: 33.15.-e, I. A. ARMIEIEVA ${ }^{1}$, V. V. ILYUSHIN ${ }^{1}$, E. A. ALEKSEEV ${ }^{1,2}$, \\ 33.20.- $\mathrm{t}$ \\ O. A. DOROVSKAYA ${ }^{1}$, L. MARGULÈS ${ }^{3}$, and R. A. MOTIYENKO ${ }^{3}$ \\ ${ }^{1}$ Institute of Radio Astronomy, National Academy of Sciences of Ukraine, \\ 4, Chervonopraporna St., Kharkiv, 61002, Ukraine \\ E-mail: ilyushin@rian.kharkov.ua \\ ${ }^{2}$ V. N. Karazin Kharkiv National University, \\ 4, Svobody Sq., Kharkiv, 61022, Ukraine \\ ${ }^{3}$ Laboratoire de Physique des Lasers, Atomes et Molécules, \\ UMR CNRS 8523, Université de Lille 1, F-59655 Villeneuve d'Ascq, France
}

\section{MILLIMETER WAVE SPECTROSCOPY OF THE GROUND, FIRST AND SECOND EXCITED TORSIONAL STATES OF ACETONE}

\begin{abstract}
Purpose: spectrum investigation of the lowest three torsional states of acetone $\left(\mathrm{CH}_{3} \mathrm{COCH}_{3}\right)$ within the frequency ranges 34-150 and 480-620 GHz.

Design/methodology/approach: New measurements were carried out using the automated millimeter wave spectrometer in the Insitute of Radio Asronomy of NASU (Kharkiv, Ukraine) and the submillimeter-wave spectrometer of PhLAM (Lille, France). The results of new measurements were fitted using a recently developed model for the molecules with two equivalent methyl rotors and $C_{2 v}$ symmetry at equilibrium (PAM_C2v_2tops program).

Findings: Analysis of the acetone molecule spectrum was carried out using the new measurements for torsion-rotation transitions in the millimeter wave range belonging to the ground, first and second excited torsional states, as well as previously published data. In addition, we performed more accurate measurements of a number of previously published lines which posed some problems for previous analysis using the same model.

Conclusions: The remeasurements have shown that the problems existed with spectrum description were caused by underestimated experimental error of the previously published data. The final fit uses 99 parameters to give an overall weighted root-meansquare deviation of 0.78 for the dataset consisting of 6233, 4868, and 4364 transitions with $J$ up to 60 and $K_{a}$ up to 35 , belonging, respectively, to the ground, first, and second excited torsional states of the acetone molecule.
\end{abstract}

Key words: acetone, millimeter wave spectrum, methyl top internal rotation

\section{Introduction}

For a long time, radio astronomy has been one of the most important sources of information about chemical composition and properties of the interstellar medium with laboratory spectroscopy being a key part of corresponding radio astronomy studies. The majority of about 190 different molecules and molecular ions detected for now in the interstellar medium have been found using the methods of microwave radio astronomy. Further progress in exploration of chemical diversity of the interstellar medium presumes not only searching for new substances but also a comprehensive account for the spectra of already detected interstellar molecules.

(C) I. A. Armieieva, V. V. Ilyushin, E. A. Alekseev,

O. A. Dorovskay, L. Margulés, and R. A. Motiyenko, 2016
In this context, acetone being a ubiquitous molecule in the interstellar medium [1-3], is of substantial interest for the radio astronomy since it has a rich and dense spectrum which is significantly complicated due to the presence of the intramolecular large amplitude motions.

Despite of more than fifty years of laboratory investigations of acetone spectrum (started in 1959 by Swalen and Costain [4]), the theoretical interpretation of the observed microwave spectrum of acetone still poses a problem. The fifty-year history of acetone spectrum studies [4-7] was documented in a series of papers by Groner and co-authors [8-10]. In these works [8-10], the analysis of rotational transitions carried out with the ERHAM program $[11,12]$, which fits the rotational levels of individual torsional states separately, without explicitly introducing a potential function. The resulting theoretical 
models [8-10] showed significant fitting problems especially in the excited torsional states $[9,10]$. A new program PAM_C2v_2tops (Principal-Axis Method for $\mathrm{C}_{2 \mathrm{v}}$ molecules with 2 tops) developed in Kharkiv for fitting the high-resolution torsion-rotation spectra of molecules with two equivalent methyl rotors and $\mathrm{C}_{2 \mathrm{v}}$ symmetry at equilibrium has been recently applied to the analysis of acetone spectrum [13]. The work [13] provided a considerable progress in fitting available experimental data on $\mathrm{CH}_{3} \mathrm{COCH}_{3}$ spectrum [8-10] and gave a firm basis for further investigations of the acetone spectrum.

In the current work, a new study of the acetone spectrum that covers frequencies within $34-150 \mathrm{GHz}$ and the range of rotational $J$ quantum number up to 60 is presented. First of all, the new study fills up the gap in the millimeter wave data on acetone spectrum clearly seen in the earlier experimental studies $[8-10]$. For the ground torsional state [8], the data within $43-141 \mathrm{GHz}$ range were absent, whereas for the first and second excited torsional states, the available data in this frequency range were rather sparse (the frequencies, respectively, of only 106 [9] and 82 [10] absorption lines were measured within $73-150 \mathrm{GHz}$ ). Our new experimental data were combined with the earlier published measurements [8-10] and fitted using a recently developed model for the molecules with two equivalent methyl rotors and $\mathrm{C}_{2 \mathrm{v}}$ symmetry at equilibrium (PAM_C2v_2tops program) [13]. In comparison with our previous analysis [13], the range of rotational quantum number $J$ has been extended up to 60 . Also, we have provided the remeasurements with higher accuracy of a number of lines in the $480-620 \mathrm{GHz}$ range (earlier measured with $200 \mathrm{kHz}$ uncertainty [8]) which posed some problems for our recent analysis of literature data using the PAM_C2v_2tops program [13]. The aforesaid problems with a group of measurements having $200 \mathrm{kHz}$ uncertainty are shown to be caused by underestimated measurement uncertainty of the old data [8].

The rest of the paper is organized as follows. Section 2 reviews experimental details of our current study. Section 3 briefly discusses the theoretical model and computer program used. Section 4 shows the results of acetone spectrum assignment and fitting. Sections 5 and 6 give some discussion and conclusions.

\section{Experimental Details}

The absorption spectrum of acetone within $34-150 \mathrm{GHz}$ range was recorded using the automated millimeter wave spectrometer of the Institute of Radio Astronomy of the National Academy of Sciences of Ukraine. This spectrometer is built according to the so-called "classical" scheme of absorption spectrometers, and its detailed description can be found in [14]. In the current study we used slightly upgraded (in comparison with [14]) version of the spectrometer. In particular, a new backward-wave oscillator (BWO) unit that covers frequency range from 34 to $52 \mathrm{GHz}$ has been put into operation. The structure of this unit is rather similar to those of other BWO units commonly applied in the spectrometer [14]. It has been found that a quasi-optic absorption cell commonly used with our spectrometer (a glass-tube of $5.6 \mathrm{~cm}$ internal diameter and $300 \mathrm{~cm}$ long) provides too high attenuation below $50 \mathrm{GHz}$, thus essentially limiting sensitivity. To solve this problem, a new waveguide absorbing cell (a copper waveguide of $10 \times 72 \mathrm{~mm}$ internal cross section and $295 \mathrm{~cm}$ long) was designed and constructed. Thus, the measurements within $34-50 \mathrm{GHz}$ were done using the new copper waveguide cell, whereas those within $50-150 \mathrm{GHz}$ were performed with the usual quasi-optic cell. All measurements were done at room temperature and with sample pressures that provided almost Doppler limited resolution (about 10 mTorr). Estimated uncertainties were 10, 30, and $100 \mathrm{kHz}$ depending on the $\mathrm{S} / \mathrm{N}$ ratio observed.

The measurements within $150-930 \mathrm{GHz}$ were performed using the spectrometer of the Laboratory of Physics of Lasers, Atoms and Molecules of Lille University, France [14]. This spectrometer is also built according to the classical scheme of absorption spectrometers. The frequency ranges 150-210, $225-315,400-630$, and $780-930 \mathrm{GHz}$ were covered with solid state multiplication sources. Frequency of the output signal of the Agilent synthesizer E8257D (12.5-17.5 GHz) was first multiplied by six and amplified by the VDI AMC-10 active sextupler providing the output power of $+14 \mathrm{dBm}$ in the $\mathrm{W}$-band range $(75-110 \mathrm{GHz})$. This power is high enough to pump passive Schottky multipliers $(\times 2, \times 3, \times 5, \times 6$, and $\times 9)$ from Virginia Diodes, Inc. in the next stage of frequency multiplication chain. The measurements were done at typical pressures of $10 \mathrm{~Pa}$ and at room temperature. Estimated uncertainties for the measured line frequencies are 30,50, 
and $100 \mathrm{kHz}$ depending on the observed $\mathrm{S} / \mathrm{N}$ ratio and frequency range.

\section{Theoretical Model}

In this study, the recently developed PAM_C2v_2tops program for fitting the high-resolution torsion-rotation spectra of molecules with two equivalent methyl rotors and $\mathrm{C}_{2 \mathrm{v}}$ symmetry at equilibrium is used [13]. This program uses the following general expression for the Hamiltonian:

$$
\begin{aligned}
& H=\frac{1}{4} \sum_{k n p q r_{1} r_{2} s_{1} S_{2} t_{1} t_{2}} B_{k n p q r_{1} r_{2} s_{1} s_{2} t_{1} t_{2}}\left\{J^{2 k} J_{z}^{n} J_{x}^{p} J_{y}^{q}\right. \\
& \times\left[p_{A}^{r_{1}} p_{B}^{r_{2}} \cos \left(3 s_{1} \alpha_{A}\right) \cos \left(3 s_{2} \alpha_{B}\right) \sin \left(3 t_{1} \alpha_{A}\right) \sin \left(3 t_{2} \alpha_{B}\right)\right. \\
& +(-1)^{(n+q)} p_{B}^{r_{1}} p_{A}^{r_{2}} \cos \left(3 s_{1} \alpha_{B}\right) \cos \left(3 s_{2} \alpha_{A}\right) \\
& \left.\times \sin \left(3 t_{1} \alpha_{B}\right) \sin \left(3 t_{2} \alpha_{A}\right)\right]+\left[(-1)^{(n+q)} \sin \left(3 t_{2} \alpha_{A}\right)\right. \\
& \times \sin \left(3 t_{1} \alpha_{B}\right) \cos \left(3 s_{2} \alpha_{A}\right) \cos \left(3 s_{1} \alpha_{B}\right) p_{A}^{r_{2}} p_{B}^{r_{1}} \\
& +\sin \left(3 t_{2} \alpha_{B}\right) \sin \left(3 t_{1} \alpha_{A}\right) \cos \left(3 s_{2} \alpha_{B}\right) \\
& \left.\left.\times \cos \left(3 s_{1} \alpha_{A}\right) p_{B}^{r_{2}} p_{A}^{r_{1}}\right] J_{y}^{q} J_{x}^{p} J_{z}^{n} J^{2 k}\right\}
\end{aligned}
$$

where the $B$ 's are fitting parameters, the quantities in brackets are quantum mechanical operators in the usual notation, and subscripts $A$ and $B$ denote the two methyl tops. The expression in Eq. (1) is written in a slightly symbolic form, in the sense that terms generated from input with $t_{1}=0$ or $t_{2}=0$ are obtained by omitting the corresponding sine functions, rather than by simply setting $t=0$ in them. The program exploits a two step diagonalization procedure. In the first step, a set of torsional computations is performed with comparatively large torsional basis set of the form $\exp \left[\left(31_{A}+\sigma_{A}\right) i \alpha_{A}\right] \exp \left[\left(31_{B}+\sigma_{B}\right) i \alpha_{B}\right]$ for each of the four different pairs of $\left(\sigma_{A}, \sigma_{B}\right)=(0,0),(0,1),(1,1)$ and $(1,2)$. In the second step, a truncated torsional basis set is used which is obtained by rejecting all but the lowest $n v t$ torsional eigenfunctions of the first step. Just as in our previous study [13], here we use the 441 basis functions in the first step and $n v t=66$ torsional eigenfunctions in the second one. More details on the used theoretical approach and corresponding computer program can be found in [13].

The opportunity to choose almost any symmetryallowed term in the Hamiltonian (by choosing an appropriate set of $k, n, p, q, r_{1}, r_{2}, s_{1}, s_{2}, t_{1}$, and $t_{2}$ integer indices in Eq. (1)) makes it possible for the user to test the efficacy of an almost unlimited number of higher-order torsion-rotation terms in the fits. The addition of new higher-order parameters requires a corresponding addition of new nomenclature, hopefully possessing some mnemonic features. In developing our nomenclature scheme we followed the general idea adopted in [15], that is to agree on a letter for a low-order term, and then add one or more subscripts to indicate higher-order corrections to this term. Our scheme looks as follows. The letter convention for the low order terms follows the spirit of [16]. We use $F, F^{\prime}, V_{3}$, etc. for loworder torsional terms in the Hamiltonian of Eq. (1). The rotational $A, B, C$ and centrifugal distortion $\Delta_{J}$, $\Delta_{J K}, \Delta_{K}$, etc. parameters have their usual notation. Terms in Eq. (1) containing a $\cos ()$ or $\cos () \cos ()$ factor are designated by the letter $V$ followed by the periodicity of the corresponding cosine factor, i.e. $V_{3}, V_{6}, V_{33}, V_{63}$, etc. Similarly, terms containing a $\sin ()$ or $\sin () \sin ()$ factor are designated by letter $V^{\prime}$ followed by the periodicity of the corresponding sine factor, i.e. $V_{33}^{\prime}, V_{63}^{\prime}$, etc. Terms containing $\cos () \sin ()$ factors are designated by the doubleletter $V V^{\prime}$, which is again followed by the periodicity of the corresponding cosine/sine factor. Addition of $J$ and $K$ subscripts to any given Hamiltonian term name has the usual meaning of adding $J^{2}$ and $J_{z}^{2}$ factors to that term (each additional $J^{2}$ or $J_{z}^{2}$ factor leads to an additional $J$ or $K$ subscript). Presence of the $J_{z}$, $J_{x}$, or $J_{y}$ factors in the term is indicated by the $z, x$, or $y$ subscripts in the term name. If the term can be characterized as multiplication of some lower-order term by $J_{x}^{2}$ or $J_{y}^{2}$, then we add an $x 2$ or $y 2$ subscript to the parent term name (the special case of addition of a $J_{z}^{2}$ factor is considered above). Multiplication by $p_{A} / p_{B}$ and $p_{A}^{2} / p_{B}^{2}$ factors (added in a way that preserves required symmetrization (see Eq. (1)) is indicated by the addition of $m$ and $m 2$ subscripts, respectively. We should distinguish two possible cases for this situation if a particular term also contains one or more $\cos ()$ or $\sin ()$ factors (the terms $\left[\left\{p_{A}, \cos \left(3 \alpha_{A}\right)\right\}-\left\{p_{B}, \cos \left(3 \alpha_{B}\right)\right\}\right] J_{z}$ and $\left[\left\{p_{A}, \cos \left(3 \alpha_{B}\right)\right\}-\left\{p_{B}, \cos \left(3 \alpha_{A}\right)\right\}\right] J_{z}$ can be considered as an example of the two possible cases where $\{A, B\}=A B+B A)$. In one case, the $p_{A}$ or $p_{A}^{2}$ factors are followed by $\cos () / \sin ()$ factors depending on $\alpha_{A}$, and the $p_{B}$ or $p_{B}^{2}$ factors are followed by $\cos () / \sin ()$ factors depending on $\alpha_{B}$ (e.g. $\left.\left[\left\{p_{A}, \cos \left(3 \alpha_{A}\right)\right\}-\left\{p_{B}, \cos \left(3 \alpha_{B}\right)\right\}\right] J_{z}\right)$. In the other case, the $p_{A}$ or $p_{A}^{2}$ factors are follo- 
wed by $\cos () / \sin ()$ factors depending on $\alpha_{B}$, and the $p_{B}$ or $p_{B}^{2}$ factors are followed by $\cos () / \sin ()$ factors depending on $\alpha_{A}$ (e.g. $\left.\left[\left\{p_{A}, \cos \left(3 \alpha_{B}\right)\right\}-\left\{p_{B}, \cos \left(3 \alpha_{A}\right)\right\}\right] J_{z}\right)$. In the first case, i.e. when the $p_{A}$ or $p_{A}^{2}$ factors are followed by $\cos () / \sin ()$ factors depending on $\alpha_{A}$ and the $p_{B}$ or $p_{B}^{2}$ factors are followed by $\cos () / \sin ()$ factors depending on $\alpha_{B}$, the corresponding subscript is placed in square brackets, i.e. $[\mathrm{m}]$ or [ $\mathrm{m} 2]$. So, if we consider the example terms given above, the first one $\left[\left\{p_{A}, \cos \left(3 \alpha_{A}\right)\right\}-\left\{p_{B}, \cos \left(3 \alpha_{B}\right)\right\}\right] J_{z}$ will get the name $V_{3[\mathrm{~m}] z}$, whereas the second one $\left[\left\{p_{A}, \cos \left(3 \alpha_{B}\right)\right\}-\left\{p_{B}, \cos \left(3 \alpha_{A}\right)\right\}\right] J_{z}$ will get the name $V_{3 m z}$. Finally, if the term can be characterized as multiplication of some lower-order term by the mixed factor $p_{A} p_{B}$, then we add an $m^{\prime}$ subscript. Numerous examples of applying these naming conventions can be found in Table 1 where parameter names are accompanied by the explicit operator forms of the corresponding Hamiltonian terms.

Table 1. Molecular parameters of acetone obtained with the PAM_C2v_2tops program from the fit of $v_{t} \leq 2$ dataset

\begin{tabular}{|c|c|c|}
\hline Operator $^{\mathrm{a}}$ & Parameter $^{b}$ & Value $^{\mathrm{c}}, \mathbf{c m}^{-1}$ \\
\hline$p_{A}^{2}+p_{B}^{2}$ & $F$ & $5.578928(15)$ \\
\hline$p_{A} p_{B}+p_{B} p_{A}$ & $F^{\prime}$ & $-0.21633(15)$ \\
\hline$(1 / 2)\left(1-\mathrm{c} 3 \alpha_{A}+1-\mathrm{c} 3 \alpha_{B}\right)$ & $V_{3}$ & $406.670(50)$ \\
\hline$(1 / 2)\left(1-\mathrm{c} 6 \alpha_{A}+1-\mathrm{c} 6 \alpha_{B}\right)$ & $V_{6}$ & $-3.813(23)$ \\
\hline$(1 / 2)\left(1-c 9 \alpha_{A}+1-c 9 \alpha_{B}\right)$ & $V_{9}$ & $-0.3363(11)$ \\
\hline$(1 / 2)\left(\mathrm{c} 3 \alpha_{A} \mathrm{c} 3 \alpha_{B}-1\right)$ & $V_{33}$ & $157.476(83)$ \\
\hline$(1 / 2) \mathrm{s} 3 \alpha_{A} \mathrm{~s} 3 \alpha_{B}$ & $V_{33}^{\prime}$ & $-164.044(28)$ \\
\hline$(1 / 2)\left(p_{A}-p_{B}\right) J_{z}$ & $4 F \rho_{z}$ & $1.309700(55)$ \\
\hline$(1 / 2)\left(p_{A}+p_{B}\right) J_{x}$ & $4 F \rho_{x}$ & $0.573554(22)$ \\
\hline$J_{z}^{2}$ & $A-(1 / 2)(B+C)$ & $0.1426987(58)$ \\
\hline$J^{2}$ & $(1 / 2)(B+C)$ & $0.2261915(11)$ \\
\hline$J_{x}^{2}-J_{y}^{2}$ & $(1 / 2)(B-C)$ & $0.06229188(80)$ \\
\hline$-J^{4}$ & $\Delta_{J}$ & $0.193305(24) \cdot 10^{-6}$ \\
\hline$-J^{2} J_{z}^{2}$ & $\Delta_{J K}$ & $-0.30999(27) \cdot 10^{-6}$ \\
\hline$-J_{z}^{4}$ & $\Delta_{K}$ & $0.72021(53) \cdot 10^{-6}$ \\
\hline$-2 J^{2}\left(J_{x}^{2}-J_{y}^{2}\right)$ & $\delta_{j}$ & $0.83472(12) \cdot 10^{-7}$ \\
\hline$-2 J_{z}^{2}\left(J_{x}^{2}-J_{y}^{2}\right)$ & $\delta_{k}$ & $0.139614(75) \cdot 10^{-6}$ \\
\hline$(1 / 2)\left(1-\mathrm{c} 3 \alpha_{A}+1-\mathrm{c} 3 \alpha_{B}\right) J^{2}$ & $V_{3 J}$ & $-0.40044(36) \cdot 10^{-3}$ \\
\hline$(1 / 2)\left(1-\mathrm{c} 3 \alpha_{A}+1-\mathrm{c} 3 \alpha_{B}\right) J_{z}^{2}$ & $V_{3 K}$ & $0.0117404(88)$ \\
\hline$(1 / 2)\left(1-\operatorname{co} 6 \alpha_{A}+1-\operatorname{cc} 6 \alpha_{B}\right) J^{2}$ & $V_{6 J}$ & $-0.775(10) \cdot 10^{-4}$ \\
\hline$(1 / 2)\left(1-\mathrm{c} 9 \alpha_{A}+1-\mathrm{c} 9 \alpha_{B}\right) J^{2}$ & $V_{9 J}$ & $0.1678(54) \cdot 10^{-4}$ \\
\hline$(1 / 2)\left(1-\mathrm{c} 6 \alpha_{A}+1-\mathrm{c} 6 \alpha_{B}\right) J_{z}^{2}$ & $V_{6 K}$ & $-0.8109(40) \cdot 10^{-4}$ \\
\hline$(1 / 2)\left(1-\mathrm{c} 3 \alpha_{A}+1-\mathrm{c} 3 \alpha_{B}\right) J_{z}^{4}$ & $V_{3 K K}$ & $-0.15753(52) \cdot 10^{-6}$ \\
\hline$(1 / 2)\left(1-\mathrm{c} 3 \alpha_{A}+1-\mathrm{c} 3 \alpha_{B}\right) J_{z}^{2} J^{2}$ & $V_{3 J K}$ & $0.1868(26) \cdot 10^{-7}$ \\
\hline$(1 / 2)\left(p_{A}^{2}+p_{B}^{2}\right) J_{z}^{2}$ & $F_{K}$ & $-0.16915(61) \cdot 10^{-4}$ \\
\hline$(1 / 2)\left(p_{A}-p_{B}\right) J_{z}^{3}$ & $\rho_{z K}$ & $-0.79732(86) \cdot 10^{-5}$ \\
\hline $\mathrm{s} 3 \alpha_{A} \mathrm{~s} 3 \alpha_{B} J_{z}^{2}$ & $V_{33 K}^{\prime}$ & $-0.26261(42) \cdot 10^{-2}$ \\
\hline$(1 / 4)\left(p_{A}+p_{B}\right)\left(J_{z}^{2} J_{x}+J_{x} J_{z}^{2}\right)$ & $\rho_{x K}$ & $0.51285(41) \cdot 10^{-5}$ \\
\hline $\mathrm{s} 3 \alpha_{A} \mathrm{~s} 3 \alpha_{B} J^{2}$ & $V_{33 J}^{\prime}$ & $0.25251(94) \cdot 10^{-3}$ \\
\hline$(1 / 2)\left(p_{A} p_{B}+p_{B} p_{A}\right) J_{z}^{2}$ & $F_{K}^{\prime}$ & $0.1996(11) \cdot 10^{-4}$ \\
\hline $\mathrm{s} 3 \alpha_{A} \mathrm{~s} 3 \alpha_{B} J_{x}^{2}$ & $V_{33 \times 2}^{\prime}$ & $0.11529(15) \cdot 10^{-2}$ \\
\hline
\end{tabular}




\begin{tabular}{|c|c|c|}
\hline Operator $^{a}$ & Parameter $^{b}$ & Value $^{\mathrm{c}}, \mathrm{cm}^{-1}$ \\
\hline$(1 / 4)\left(p_{A}-p_{B}\right)\left(J_{z} J_{x}^{2}+J_{x}^{2} J_{z}\right)$ & $\rho_{z x 2}$ & $-0.25667(42) \cdot 10^{-5}$ \\
\hline$(1 / 2)\left(\mathrm{c} 3 \alpha_{A}+\mathrm{c} 3 \alpha_{B}\right) J_{x}^{2}$ & $V_{3 x 2}$ & $0.55537(43) \cdot 10^{-2}$ \\
\hline$(1 / 2)\left(p_{A}+p_{B}\right) J_{x} J^{2}$ & $\rho_{x J}$ & $-0.9930(23) \cdot 10^{-6}$ \\
\hline$(1 / 2)\left(p_{A}^{2}+p_{B}^{2}\right) J^{2}$ & $F_{J}$ & $-0.53425(79) \cdot 10^{-5}$ \\
\hline$(1 / 2)\left(p_{A}^{3}-p_{B}^{3}\right) J_{z}$ & $\rho_{z m 2}$ & $0.9401(24) \cdot 10^{-4}$ \\
\hline$(1 / 2)\left(\mathrm{s} 6 \alpha_{A} \mathrm{~s} 3 \alpha_{B}+\mathrm{s} 6 \alpha_{B} \mathrm{~s} 3 \alpha_{A}\right)$ & $V_{63}^{\prime}$ & $-4.4567(60)$ \\
\hline $\mathrm{c} 3 \alpha_{A} \mathrm{c} 3 \alpha_{B} J_{x}^{2}$ & $V_{33 \times 2}$ & $-0.13594(25) \cdot 10^{-2}$ \\
\hline $\mathrm{c} 3 \alpha_{A} \mathrm{c} 3 \alpha_{B} J_{z}^{2}$ & $V_{33 K}$ & $0.15222(21) \cdot 10^{-2}$ \\
\hline$(1 / 2)\left(p_{A}^{3}+p_{B}^{3}\right) J_{x}$ & $\rho_{x m 2}$ & $-0.3624(14) \cdot 10^{-4}$ \\
\hline$(1 / 4)\left(\left\{p_{B}, \mathrm{~s} 3 \alpha_{A}\right\}-\left\{p_{A}, \mathrm{~s} 3 \alpha_{B}\right\}\right) J_{y}$ & $V_{3 m y}^{\prime}$ & $0.3301(11) \cdot 10^{-2}$ \\
\hline$(1 / 2)\left(p_{A}+p_{B}\right) J_{x}^{3}$ & $\rho_{x x 2}$ & $-0.23051(24) \cdot 10^{-5}$ \\
\hline$(1 / 4)\left(\mathrm{s} 3 \alpha_{A}+\mathrm{s} 3 \alpha_{B}\right)\left(J_{z} J_{y}+J_{y} J_{z}\right)$ & $V_{3 z y}^{\prime}$ & $-0.6493(21) \cdot 10^{-3}$ \\
\hline$(1 / 4)\left(\left\{p_{A}, \mathrm{~s} 3 \alpha_{A}, \mathrm{~s} 3 \alpha_{B}\right\}-\left\{p_{B}, \mathrm{~s} 3 \alpha_{B}, \mathrm{~s} 3 \alpha_{A}\right\}\right) J_{z}$ & $V_{33[m] z}^{\prime}$ & $-0.015259(73)$ \\
\hline$(1 / 2) \mathrm{s} 3 \alpha_{A} \mathrm{~s} 3 \alpha_{B}\left(J_{z}^{2} J_{x}^{2}+J_{x}^{2} J_{z}^{2}\right)$ & $V_{33 K x 2}^{\prime}$ & $0.5654(41) \cdot 10^{-7}$ \\
\hline$(1 / 4)\left(\left\{p_{B}, \mathrm{c} 3 \alpha_{A}\right\}-\left\{p_{A}, \mathrm{c} 3 \alpha_{B}\right\}\right) J_{z}$ & $V_{3 m z}$ & $0.019945(63)$ \\
\hline$(1 / 2)\left(\mathrm{s} 9 \alpha_{A} \mathrm{~s} 3 \alpha_{B}+\mathrm{s} 9 \alpha_{B} \mathrm{~s} 3 \alpha_{A}\right)$ & $V_{93}^{\prime}$ & $-1.3508(48)$ \\
\hline$(1 / 4)\left(\left\{p_{A}^{2}, p_{B}\right\}-\left\{p_{B}^{2}, p_{A}\right\}\right) J_{z}$ & $\rho_{z m^{\prime}}$ & $0.587(10) \cdot 10^{-4}$ \\
\hline$(1 / 4)\left(\left\{p_{B}, \mathrm{c} 3 \alpha_{A}\right\}+\left\{p_{A}, \mathrm{c} 3 \alpha_{B}\right\}\right) J_{x}$ & $V_{3 m x}$ & $0.3751(27) \cdot 10^{-2}$ \\
\hline$(1 / 2)\left(p_{A}^{2} p_{B}^{2}+p_{B}^{2} p_{A}^{2}\right)$ & $F_{m^{\prime}}^{\prime}$ & $-0.1641(31) \cdot 10^{-3}$ \\
\hline$(1 / 2)\left(\mathrm{c} 6 \alpha_{A} \mathrm{c} 3 \alpha_{B}+\mathrm{c} 6 \alpha_{B} \mathrm{c} 3 \alpha_{A}\right) J^{2}$ & $V_{63 J}$ & $-0.13937(74) \cdot 10^{-3}$ \\
\hline$(1 / 4)\left(\left\{p_{A}, \mathrm{c} 3 \alpha_{A}\right\}-\left\{p_{B}, \mathrm{c} 3 \alpha_{B}\right\}\right) J_{z}$ & $V_{3[\mathrm{~m}] z}$ & $-0.075884(96)$ \\
\hline$(1 / 4)\left(\left\{p_{B}^{2}, \mathrm{~s} 6 \alpha_{A}, \mathrm{~s} 3 \alpha_{B}\right\}+\left\{p_{A}^{2}, \mathrm{~s} 6 \alpha_{B}, \mathrm{~s} 3 \alpha_{A}\right\}\right)$ & $V_{63 m 2}^{\prime}$ & $0.02126(39)$ \\
\hline$(1 / 4)\left(\left\{p_{B}, \mathrm{~s} 3 \alpha_{A}\right\}-\left\{p_{A}, \mathrm{~s} 3 \alpha_{B}\right\}\right)\left(J_{x}^{2} J_{y}+J_{y} J_{x}^{2}\right)$ & $V_{3 m y x 2}^{\prime}$ & $0.1236(31) \cdot 10^{-6}$ \\
\hline$(1 / 2)\left(\mathrm{c} 6 \alpha_{A}+\mathrm{c} 6 \alpha_{B}\right) J_{x}^{2}$ & $V_{6 x 2}$ & $0.4935(40) \cdot 10^{-4}$ \\
\hline$(1 / 4)\left(\left\{p_{A}, \mathrm{~s} 6 \alpha_{A}, \mathrm{~s} 3 \alpha_{B}\right\}+\left\{p_{B}, \mathrm{~s} 6 \alpha_{B}, \mathrm{~s} 3 \alpha_{A}\right\}\right) J_{x}$ & $V_{63[m] x}^{\prime}$ & $-0.013394(42)$ \\
\hline$J^{6}$ & $H_{J}$ & $0.4190(25) \cdot 10^{-12}$ \\
\hline$J^{4} J_{z}^{2}$ & $H_{J K}$ & $-0.376(10) \cdot 10^{-12}$ \\
\hline$J^{2} J_{z}^{4}$ & $H_{K J}$ & $-0.4964(29) \cdot 10^{-11}$ \\
\hline$J_{z}^{6}$ & $H_{K}$ & $0.7785(29) \cdot 10^{-11}$ \\
\hline $2\left(J_{x}^{2}-J_{y}^{2}\right) J^{4}$ & $h_{j}$ & $0.2095(13) \cdot 10^{-12}$ \\
\hline$\left\{J_{z}^{4},\left(J_{x}^{2}-J_{y}^{2}\right)\right\}$ & $h_{k}$ & $0.2891(17) \cdot 10^{-11}$ \\
\hline$(1 / 4)\left(\mathrm{s} 6 \alpha_{A} \mathrm{~s} 3 \alpha_{B}-\mathrm{s} 6 \alpha_{B} \mathrm{~s} 3 \alpha_{A}\right)\left(J_{z} J_{x}+J_{x} J_{z}\right)$ & $V_{63 z x}^{\prime}$ & $-0.13304(47) \cdot 10^{-2}$ \\
\hline$(1 / 4)\left(\left\{p_{A}, \mathrm{c} 3 \alpha_{A}\right\}-\left\{p_{B}, \mathrm{c} 3 \alpha_{B}\right\}\right)\left(J_{z} J_{x}^{2}+J_{x}^{2} J_{z}\right)$ & $V_{3[m] z x 2}$ & $-0.6402(55) \cdot 10^{-6}$ \\
\hline$(1 / 4)\left(\mathrm{s} 3 \alpha_{A}-\mathrm{s} 3 \alpha_{B}\right)\left(J_{x} J_{y}+J_{y} J_{x}\right)$ & $V_{3 x y}^{\prime}$ & $-0.25570(40) \cdot 10^{-2}$ \\
\hline$(1 / 2)\left(p_{A}^{4}+p_{B}^{4}\right)$ & $F_{m 2}$ & $-0.59013(78) \cdot 10^{-3}$ \\
\hline$(1 / 2)\left(\mathrm{c} 3 \alpha_{A}+\mathrm{c} 3 \alpha_{B}\right) J_{x}^{4}$ & $V_{3 \times 2 \times 2}$ & $-0.25865(93) \cdot 10^{-7}$ \\
\hline$(1 / 4)\left(\left\{p_{B}, \mathrm{~s} 6 \alpha_{A}\right\}-\left\{p_{A}, \mathrm{~s} 6 \alpha_{B}\right\}\right) J_{y}$ & $V_{6 m y}^{\prime}$ & $0.2163(24) \cdot 10^{-2}$ \\
\hline$(1 / 4)\left(\left\{p_{A}^{2}, p_{B}\right\}+\left\{p_{B}^{2}, p_{A}\right\}\right) J_{x}$ & $\rho_{x m^{\prime}}$ & $0.7136(68) \cdot 10^{-4}$ \\
\hline$(1 / 2)\left(\mathrm{c} 6 \alpha_{A} \mathrm{c} 3 \alpha_{B}-1+\mathrm{c} 6 \alpha_{B} \mathrm{c} 3 \alpha_{A}-1\right)$ & $V_{63}$ & $6.289(39)$ \\
\hline $\mathrm{c} 6 \alpha_{A} \mathrm{c} 6 \alpha_{B}-1$ & $V_{66}$ & $-1.617(10)$ \\
\hline$(1 / 4)\left(\left\{p_{B}, \mathrm{~s} 6 \alpha_{A}, \mathrm{~s} 3 \alpha_{B}\right\}+\left\{p_{A}, \mathrm{~s} 6 \alpha_{B}, \mathrm{~s} 3 \alpha_{A}\right\}\right) J_{x}$ & $V_{63 m x}^{\prime}$ & $0.8502(32) \cdot 10^{-2}$ \\
\hline$(1 / 4)\left(\left\{p_{B}, \mathrm{c} 6 \alpha_{A}\right\}+\left\{p_{A}, \mathrm{c} 6 \alpha_{B}\right\}\right) J_{x}$ & $V_{6 m x}$ & $0.41390(96) \cdot 10^{-2}$ \\
\hline$(1 / 4)\left(\mathrm{s} 6 \alpha_{A}+\mathrm{s} 6 \alpha_{B}\right)\left(J_{z} J_{y}+J_{y} J_{z}\right)$ & $V_{6 z y}^{\prime}$ & $-0.2317(20) \cdot 10^{-3}$ \\
\hline
\end{tabular}




\begin{tabular}{|c|c|c|}
\hline Operator $^{\mathrm{a}}$ & Parameter $^{b}$ & Value $^{\mathrm{c}}, \mathrm{cm}^{-1}$ \\
\hline $\begin{array}{c}(1 / 4)\left(\left\{p_{B}, \mathrm{~s} 6 \alpha_{A}\right\}-\left\{p_{A}, \mathrm{~s} 6 \alpha_{B}\right\}\right)\left(J_{z}^{2} J_{y}+J_{y} J_{z}^{2}\right) \\
(1 / 2)\left(\mathrm{s} 6 \alpha_{A} \mathrm{~s} 3 \alpha_{B}+\mathrm{s} 6 \alpha_{B} \mathrm{~s} 3 \alpha_{A}\right) J^{2} \\
(1 / 4)\left(\mathrm{c} 3 \alpha_{A}+\mathrm{c} 3 \alpha_{B}\right)\left(J_{z}^{2} J_{x}^{2}+J_{x}^{2} J_{z}^{2}\right) \\
(1 / 4)\left(\left\{p_{A}, p_{B}, \mathrm{c} 3 \alpha_{A}, \mathrm{c} 3 \alpha_{B}\right\}+\left\{p_{B}, p_{A}, \mathrm{c} 3 \alpha_{B}, \mathrm{c} 3 \alpha_{A}\right\}\right) J_{x}^{2} \\
(1 / 2)\left(p_{A}+p_{B}\right) J_{x}^{3} J^{2} \\
(1 / 4)\left(\left\{p_{A}^{3}, \mathrm{~s} 3 \alpha_{A}, \mathrm{~s} 3 \alpha_{B}\right\}+\left\{p_{B}^{3}, \mathrm{~s} 3 \alpha_{B} \mathrm{~s} 3 \alpha_{A}\right\}\right) J_{x} \\
(1 / 4)\left(\mathrm{c} 6 \alpha_{B} \mathrm{~s} 3 \alpha_{A}-\mathrm{c} 6 \alpha_{A} \mathrm{~s} 3 \alpha_{B}\right)\left(J_{x} J_{y}+J_{y} J_{x}\right) \\
(1 / 2)\left(p_{A}^{3} p_{B}^{3}+p_{B}^{3} p_{A}^{3}\right) \\
(1 / 4)\left(\left\{p_{A}, \mathrm{c} 3 \alpha_{A}\right\}-\left\{p_{B}, \mathrm{c} 3 \alpha_{B}\right\}\right) J_{z}^{3} \\
(1 / 2)\left(p_{A}^{3} p_{B}^{2}-p_{B}^{3} p_{A}^{2}\right) J_{z} \\
(1 / 4)\left(\mathrm{s} 3 \alpha_{A}-\mathrm{s} 3 \alpha_{B}\right)\left(J_{z}^{2} J_{x} J_{y}+J_{y} J_{x} J_{z}^{2}\right) \\
(1 / 4)\left(p_{A}+p_{B}\right)\left(J_{z}^{2} J_{x}+J_{x} J_{z}^{2}\right) J^{2} \\
(1 / 2)\left(p_{A}^{2}+p_{B}^{2}\right) J_{x}^{2} \\
(1 / 4)\left(\left\{p_{B}, \mathrm{c} 3 \alpha_{A}\right\}-\left\{p_{A}, \mathrm{c} 3 \alpha_{B}\right\}\right)\left(J_{z} J_{x}^{2}+J_{x}^{2} J_{z}\right) J^{2} \\
(1 / 4)\left(p_{A}^{3} p_{B}-p_{B}^{3} p_{A}\right)\left(J_{z} J_{x}+J_{x} J_{z}\right) J^{2} \\
(1 / 4)\left(\left\{p_{A}, \mathrm{~s} 3 \alpha_{A}, \mathrm{~s} 3 \alpha_{B}\right\}-\left\{p_{B}, \mathrm{~s} 3 \alpha_{B}, \mathrm{~s} 3 \alpha_{A}\right\}\right)\left(J_{z} J_{x}^{2}+J_{x}^{2} J_{z}\right) \\
(1 / 4)\left(\mathrm{s} 3 \alpha_{A}-\mathrm{s} 3 \alpha_{B}\right)\left(J_{x}^{3} J_{y}+J_{y} J_{x}^{3}\right) \\
(1 / 4)\left(\left\{p_{B}, \mathrm{c} 3 \alpha_{A}\right\}-\left\{p_{A}, \mathrm{c} 3 \alpha_{B}\right\}\right) J_{z}^{5} \\
(1 / 4)\left(p_{A}^{2}-p_{B}^{2}\right)\left(J_{z} J_{x}+J_{x} J_{z}\right) \\
(1 / 4)\left(\left\{p_{A}, \mathrm{~s} 3 \alpha_{A}, \mathrm{~s} 3 \alpha_{B}\right\}+\left\{p_{B}, \mathrm{~s} 3 \alpha_{B}, \mathrm{~s} 3 \alpha_{A}\right\}\right) J_{x}^{3} \\
(1 / 2)\left(p_{A} p_{B}+p_{B} p_{A}\right) J_{z}^{4} \\
(1 / 4)\left(\left\{p_{A}, p_{B}, \mathrm{c} 3 \alpha_{A}, \mathrm{c} 3 \alpha_{B}\right\}+\left\{p_{B}, p_{A}, \mathrm{c} 3 \alpha_{B}, \mathrm{c} 3 \alpha_{A}\right\}\right) J_{z}^{2} \\
\mathrm{~s} 3 \alpha_{A} \mathrm{~s} 3 \alpha_{B} J_{x}^{2} J^{2} \\
(1 / 4)\left(\left\{p_{A}, p_{B}, \mathrm{c} 3 \alpha_{A}, \mathrm{c} 3 \alpha_{B}\right\}+\left\{p_{B}, p_{A}, \mathrm{c} 3 \alpha_{B}, \mathrm{c} 3 \alpha_{A}\right\}\right) \\
(1 / 4)\left(\left\{p_{B}, \mathrm{c} 3 \alpha_{A}\right\}-\left\{p_{A}, \mathrm{c} 3 \alpha_{B}\right\}\right) J_{z} J^{2}\end{array}$ & $\begin{array}{c}V_{6 m y K K}^{\prime} \\
V_{63 J}^{\prime} \\
V_{3 K x 2} \\
V_{33 m^{\prime} x 2} \\
\rho_{x x 2 J} \\
V_{33 m x m 2}^{\prime} \\
V V_{63 x y}^{\prime} \\
F_{m^{\prime} m^{\prime}}^{\prime} \\
V_{3[m] z K} \\
\rho_{z m^{\prime} m^{\prime}} \\
V_{3 x y K}^{\prime} \\
\rho_{x J K} \\
F_{x 2} \\
V_{3 m z J K} \\
F_{m^{\prime} z x J} \\
V_{33[m] x x 2}^{\prime} \\
V_{3 x y x 2}^{\prime} \\
V_{3 m z K K} \\
F_{z x} \\
V_{33[m] x x 2}^{\prime} \\
F_{K K}^{\prime} \\
V_{33 m^{\prime} K} \\
V_{33 J x 2}^{\prime} \\
V_{33 m^{\prime}} \\
V_{3 m z J}\end{array}$ & $\begin{array}{c}0.1958(24) \cdot 10^{-6} \\
-0.6895(86) \cdot 10^{-4} \\
0.6092(61) \cdot 10^{-7} \\
0.7289(71) \cdot 10^{-5} \\
0.902(19) \cdot 10^{-11} \\
0.7290(62) \cdot 10^{-4} \\
-0.2529(25) \cdot 10^{-3} \\
0.583(11) \cdot 10^{-5} \\
0.14310(65) \cdot 10^{-5} \\
-0.929(13) \cdot 10^{-6} \\
0.1746(26) \cdot 10^{-6} \\
-0.2298(26) \cdot 10^{-10} \\
-0.3014(24) \cdot 10^{-5} \\
-0.684(17) \cdot 10^{-11} \\
-0.2418(33) \cdot 10^{-11} \\
0.1559(89) \cdot 10^{-6} \\
0.4951(70) \cdot 10^{-7} \\
-0.1587(26) \cdot 10^{-10} \\
0.3244(49) \cdot 10^{-5} \\
0.1346(35) \cdot 10^{-6} \\
0.3607(48) \cdot 10^{-9} \\
0.6596(52) \cdot 10^{-5} \\
-0.638(10) \cdot 10^{-8} \\
0.01665(82) \\
0.554(31) \cdot 10^{-7}\end{array}$ \\
\hline
\end{tabular}

a Operator which is multiplied by the parameter in the program, where $\{A, B\} \equiv A B+B A, \quad\{A, B, C\} \equiv A B C+C B A$, $\{\mathrm{A}, \mathrm{B}, \mathrm{C}, \mathrm{D}\} \equiv \mathrm{ABCD}+\mathrm{DCBA}, \quad \mathrm{c} x=\cos x$ and $\mathrm{s} x=\sin x$.

${ }^{\mathrm{b}}$ For parameter nomenclature see discussion in the text.

${ }^{\mathrm{c}}$ Statistical uncertainties (parenthesized) are shown as one standard uncertainty in the last two digits.

\section{Spectral Analysis and Fit}

We started our analysis from the results of [13] where the weighted standard deviation of 0.94 was achieved for the dataset consisting of 13 FIR (Far Infrared) line frequencies and 1720 microwave transitions with $J \leq 38$ and $K_{a} \leq 21$ that was fitted using 40 parameters of the Hamiltonian model which assumes two equivalent methyl rotors and $\mathrm{C}_{2 \mathrm{v}}$ symmetry at equilibrium (PAM_C2v_2tops program). Assigning and fitting the new data using the PAM_C2v_2tops program proceeded in a fairly conventional iterative way by going up in frequency, with assignments for all three torsional states done simultaneously since our previous study [13] provided rather good starting predictions. As a result, about 10000 new line frequencies within 34-150 GHz range have been added to the initial dataset [13]. Furthermore, we remeasured a number of lines in the $480-620 \mathrm{GHz}$ range which were previously obtained with $200 \mathrm{kHz}$ uncertainty [8]. These lines have posed some problem for our recent analysis of literature data using the PAM_C2v_2tops program [13]. The comparison of the remeasured transition frequencies with the old data [8] will be discussed in more detail in Section 5. Here, it should be mentioned that all data with $200 \mathrm{kHz}$ uncertainty in the $480-620 \mathrm{GHz}$ range from [8] were replaced in the final dataset with the new more accurate measurements obtained in our current study. 
The final dataset consists of 12128 microwave line frequencies and 7 FIR band origins. Due to clustering in the acetone spectrum and occasional blending these 12128 line frequencies correspond to 15465 rotational transitions in the fit. In comparison with our previous study [13], the dataset was significantly enlarged, and more than 10000 new line frequencies were added to the analysis. Table 2 gives an overview of the $\left(\sigma_{A}, \sigma_{B}\right)=(0,0),(0,1),(1,1)$ and $(1,2)$ symmetry species coverage in $G_{9}$, as well as the $J, K_{a}$, and $v$ quantum number coverage of the dataset used in the final fits of this paper. Comparison of Table 2 with Table 4 in [13], where analogous overview of the previous dataset is given, shows that the range of rotational quantum numbers was most significantly expanded in the $K_{a}$ quantum number (especially for the second excited torsional state $\left.v_{17}\right)$.

A fit achieving a weighted root-mean-square (rms) deviation of 0.78 for 12128 microwave and 7 FIR line frequencies with 99 parameters included in the model was chosen as our "best fit" for this paper. The 99 molecular parameters obtained from this fit are given in Table 1. Although the number of parameters in the model was significantly increased (99 here against 40 in [13]), our current study shows further improvement of the lines/parameter ratio in comparison with the previous results $(\approx 122$ lines/parameter here against $\approx 43$ lines per adjustable parameter in [13] and $\approx 19$ lines/parameter in [8-10]). The quality of the fit chosen as our "best fit" for this paper can be seen from the overview of Table 2. The overall weighted standard deviation of 0.78 , as well as the fact that all data groups are fit within their experimental uncertainties (see middle part of Table 2 where the data are grouped by measurement uncertainty), seems to be completely satisfactory.

\section{Discussion}

A significant increasing (almost 2.5 times) of the number of parameters in the model in comparison with our previous work [13] is caused in our opinion by expanded rotational quantum number range involved in the analysis (which is especially evident for the second excited torsional state, see Table 2). Also, our new data provide a higher level of accuracy of measurements than that observed in the previous dataset [13]. Indeed, in [13], the dominating group of data belongs to the category with $100 \mathrm{kHz}$ uncertainty (see Table 4 in [13]), whereas in the current fit, the category with $10 \mathrm{kHz}$ measurement uncertainty is dominant (see Table 2 here). Thus, on average we

Table 2. Overview of the dataset and fit quality

\begin{tabular}{|c|c|c|c|c|c|c|c|c|c|}
\hline \multicolumn{3}{|c|}{ By symmetry } & \multicolumn{2}{c|}{ By measurement uncertainty } & \multicolumn{3}{c|}{ By torsional state } \\
\hline$\left(\boldsymbol{\sigma}_{\boldsymbol{A}}, \boldsymbol{\sigma}_{\boldsymbol{B}}\right)^{\mathbf{a}}$ & $\#^{\mathbf{b}}$ & $\mathbf{r m s}^{\mathbf{c}}, \mathbf{k H z}$ & $\mathbf{U n c}^{\mathbf{d}}, \mathbf{k H z}$ & $\#^{\mathbf{e}}$ & $\mathbf{r m s}^{\mathbf{c}}, \mathbf{k H z}$ & $\boldsymbol{v}^{\mathbf{f}}$ & $\#^{\mathbf{b}}$ & $\mathbf{r m s}^{\mathbf{c}}, \mathbf{k H z}$ & $\boldsymbol{J}, \boldsymbol{K}_{\boldsymbol{a}}^{\mathbf{g}}$ \\
\hline$(0,0)$ & 3819 & 42.73 & 4 & 14 & 1.42 & $\mathrm{GS}$ & 6233 & 43.41 & 60,35 \\
$(0,1)$ & 4912 & 37.91 & 8 & 20 & 3.72 & $v_{12}$ & 4868 & 47.42 & 52,32 \\
$(1,1)$ & 3313 & 52.48 & 10 & 6793 & 8.30 & $v_{17}$ & 4364 & 45.95 & 43,29 \\
$(1,2)$ & 3421 & 50.57 & 20 & 43 & 11.77 & & & \\
& & & 30 & 2805 & 19.39 & & & \\
& & & 50 & 755 & 31.85 & & & \\
& & & 100 & 1588 & 82.96 & & & & \\
& & & 150 & 95 & 49.42 & & & & \\
\end{tabular}

\footnotetext{
${ }^{\text {a }}$ Symmetry species in $\mathrm{G}_{9}$ symmetry group.

${ }^{\mathrm{b}}$ Number of transitions of each symmetry species included in the least-squares fit of this paper, with a weighted standard deviation of 0.78. (Parameters from this fit are given in Table 1.) Only rotational transitions are considered in this table. The rms deviation of the 7 vibrational frequencies from Table 2 in [16] is $0.372 \mathrm{~cm}^{-1}$. Note, that due to blending, 15465 rotational transitions correspond to 12128 measured line frequencies in the fit.

${ }^{c}$ Root-mean-square (rms) deviation for each data group.

${ }^{\mathrm{d}}$ Estimated measurement uncertainties for each data group.

e Number of measured line frequencies in each data group.

${ }^{f} \mathrm{GS}$ represents the ground torsional state; $v_{12}$ and $v_{17}$ represent excited torsional states with $v_{12}=1$ and $v_{17}=1$, respectively.

${ }^{\mathrm{g}}$ The maximum values of $J$ and $K_{a}$ for transitions in each torsional state included in the fit.
} 
need now a theoretical model which should be adequate at the level being by order of magnitude higher than in the previous study [13]. A higher level of accuracy will certainly require an inclusion of additional higher order terms to take into account more subtle torsion-rotation effects becoming evident at this increased level of accuracy. At the same time, it should be noted that the current number of parameters (even with an increased range of quantum numbers and significantly improved average level of measurement uncertainty) is still less than the number of parameters (105) employed in [8-10] to fit earlier available smaller set of data. Finally, we would like to mention that despite the fact that in acetone we have two large amplitude torsional motions, the number of parameters used in our model is comparable with that used to fit spectra of some other molecules with only one torsional large amplitude motion [15, 17, 18]. From the arguments given above we can conclude that the number of terms used in the model is physically sound and fairly well balanced with the amount of available experimental data.

It is interesting to compare the values of the low order torsion parameters with the results of the previous study [13] because of a rather broad variation of $V_{3}$ and $V_{33}$ values among different fits to near experimental error (weighted rms deviation $\approx 1$ ) observed in [13]. As was mentioned in [13], changes in the $V_{3}$ and $V_{33}$ values in the fits were always triggered by changes in the parameter set, and therefore we anticipated that an addition of new terms to the model will affect low order torsion parameters. Comparison of the $V_{3}, V_{33}, V_{33}^{\prime}, F, F^{\prime}$, and $\rho$ values from Table 1 with the corresponding values from Table 5 in [13] shows that variations of the parameter values are well above the confidence intervals from the previous study [13] with the largest changes observed namely for $V_{3}, V_{33}$, and $V_{33}^{\prime}$ parameters. In our opinion, these large changes in low order parameter values are caused by significant changes in the model and dataset, as well as by the correlation between $V_{3}$ and $V_{33}$ which is a known issue discussed in some detail by Groner and co-authors $[19,20]$. At the same time, it should be noted that in our current fitting attempts we have observed significantly narrower variation of $V_{3}$ and $V_{33}$ values among the exploratory fits to near experimental error. Therefore we think that our new experimental data gave an opportunity to put tighter constraints on the highly correlated low order torsional parameters. We hope that further experimental investigation of the acetone spectrum, especially an addition of the high-resolution data on acetone torsional bands, will allow significant reducing of the correlation between low order torsional parameters in our model, thus providing an opportunity of benchmark comparison with ab initio calculations.

One of the issues remained unresolved in our recent analysis of literature data using the PAM_C2v_2tops program [13] is the situation with the $200 \mathrm{kHz}$ data group, the only one not fit within experimental error in [13]. This group of data represented the transitions with the highest $K_{a}$ quantum numbers in the fit, and therefore a reasonable question had emerged whether these fitting problems were due to some limitations of the theoretical model or were of pure experimental nature. In the previous work [13], the assumption was made that the systematic errors in the early FASSST measurements (the data [8] were recorded using an early version of the FASSST spectrometer at Ohio State University) is the cause of the fitting problems in the $200 \mathrm{kHz}$ uncertainty data group. To check this assumption, we have remeasured the line frequencies within $480-620 \mathrm{GHz}$ range for the $200 \mathrm{kHz}$ data group in [8] using the records of the acetone spectrum obtained in PhLAM laboratory (Lille, France). In contrast to the FASSST spectrometer at Ohio State University, the Lille spectrometer is a synthesizer based one and therefore it provides much better frequency calibration of measurements. Differences between transition frequencies from [8] and our new remeasured values for the same lines are shown in Fig. 1. It is evident that a number of differences up to $1 \mathrm{MHz}$ are observed. About $30 \%$ of the lines have the differences between 200 and $400 \mathrm{kHz}$, and about $20 \%$ of the lines have the differences above $400 \mathrm{kHz}$, i.e. well above experimental uncertainty stated for these lines in [8]. At the same time, the new remeasured frequencies are fitted within experimental error (mainly 30 or $50 \mathrm{kHz}$ ), whereas the earlier measured frequencies are not. Thus, we can conclude that systematic errors in the early FASSST measurements were indeed the cause of our fitting problems [13] in the $200 \mathrm{kHz}$ uncertainty data group [8]. As was already mentioned, in our current fitting attempts all these data were replaced by the new more accurate measurements obtained with the use of the Lille spectrometer. 

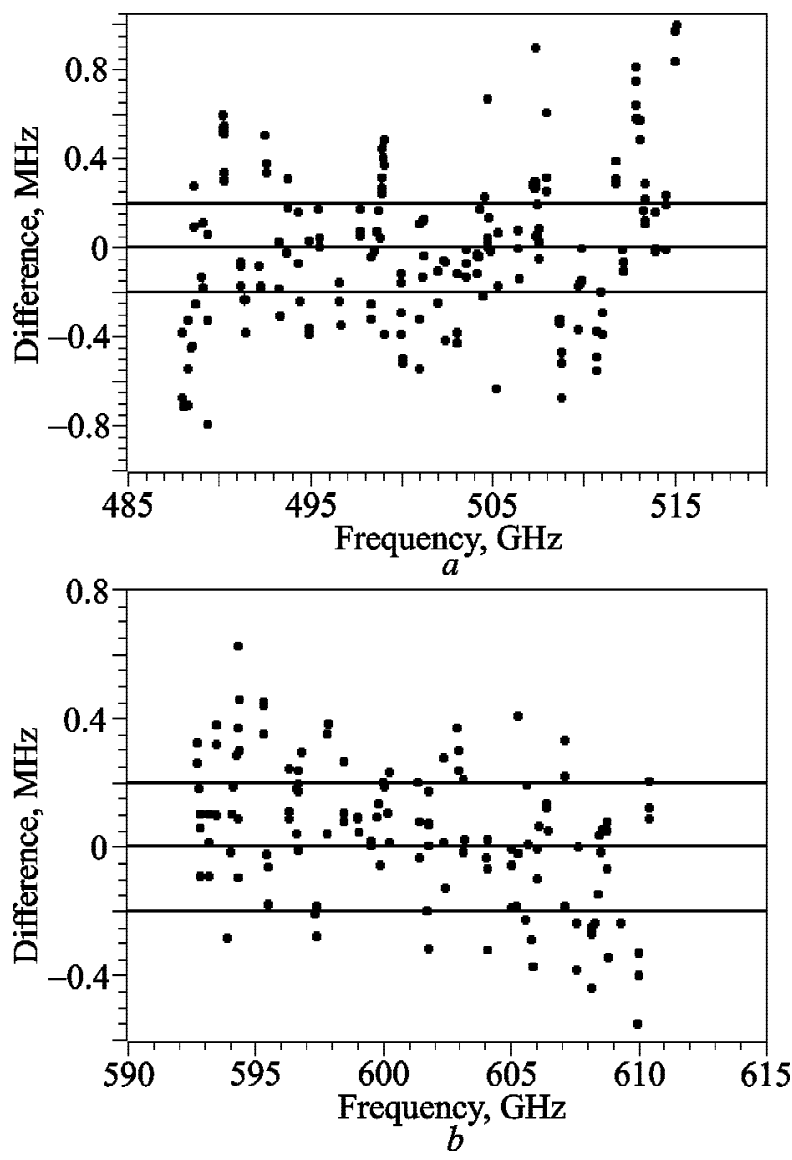

Fig. 1. Differences between remeasured in the current work transition frequencies and those observed in [8] for the $200 \mathrm{kHz}$ uncertainty group of data. Fig. 1(a) represents $485-520 \mathrm{GHz}$ frequency range, Fig. 1(b) represents 590-615 GHz frequency range

\section{Conclusions}

We have presented a new study of rotational spectra in the lowest three torsional states of acetone $\mathrm{CH}_{3} \mathrm{COCH}_{3}$ employing the model developed for molecules with two equivalent methyl rotors and $\mathrm{C}_{2 \mathrm{v}}$ symmetry at equilibrium that makes use of an explicit twodimensional potential function. In the current study, the dataset available in the literature was augmented by the new measurements in the millimeter wave range as well as by remeasurement of a number of transitions in the submillimeter wave range. About 10000 new line frequencies were added to the analysis. The set of 99 Hamiltonian parameters, which fits within the experimental error the dataset of 12128 microwave and 7 FIR acetone line frequencies, was obtained. The experimental dataset sample all symmetry species in $G_{36}$ of the ground, $v_{12}=1$, and $v_{17}=1$ torsional states and covers the frequency range from 8 to $610 \mathrm{GHz}$. It is shown that previously reported problems of our analysis of literature data using the PAM_C2v_2tops program [13] were caused by underestimated experimental uncertainty of the old data [8]. Our remeasurements have allowed to resolve these problems. The obtained results provide a firm basis for further investigations of the submillimeter wave spectrum of acetone and for reliable predictions of the acetone spectrum in the millimeter wave range for the needs of radio astronomy.

\section{Asknowledgements}

This work was partially done under support of the Ukrainian-French CNRS-PICS 6051 project.

\section{REFERENCES}

1. Combes F., Gerin M., Wooten A., Wlodarczak G., Clausset F., and Encrenaz P. J. Acetone in interstellar space // Astron. Astrophys. - 1987. - Vol. 180, No. 1-2. P. L13-L16.

2. Snyder L. E., Lovas F. J., Mehringer D. M., Miao N. Y., Kuan Y.-J., Hollis J. M., and Jewell P. R. Confirmation of interstellar acetone // Astrophys. J. - 2002. - Vol. 578, No. 1. - P. 245-255. DOI: 10.1086/342273

3. Friedel D. N., Snyder L. E., Remijan A. J., and Turner B. E. Detection of interstellar acetone toward the Orion-KL hot core // Astrophys. J. - 2005. - Vol. 632, No. 1. P. L95-L98. DOI: 10.1086/497986

4. Swalen J. D. and Costain C. C. Internal rotation in molecules with two internal rotors: microwave spectrum of acetone // J. Chem. Phys. - 1959. - Vol. 31. - P. 1562-1574. DOI: $10.1063 / 1.1730653$

5. Nelson R., and Pierce L. Microwave spectrum, structure, and barrier to internal rotation of acetone // J. Mol. Spectrosc. - 1965. - Vol. 18, Is. 3. - P. 344-352. DOI: 10.1016/ 0022-2852(65)90144-X

6. Vacherand J. M., van Eijck B. P., Burie J., and Demaison J. The rotational spectrum of acetone: internal rotation and centrifugal distortion analysis // J. Mol. Spectrosc. 1986. - Vol. 118, Is. 2. - P. 355-362. DOI: 10.1016/ 0022-2852(86)90175-X

7. Oldag F. and Sutter D. H. The rotational zeeman effect in acetone, its g-tensor, its magnetic susceptibility anisotropies and its molecular electric quadrupole moment tensor; a high resolution microwave fourier transform study // Z. Naturforch. A. - 1992. - Vol. 47, Is. 3. - P. 527-532. DOI: $10.1515 /$ zna-1992-0315

8. Groner P., Albert S., Herbst E., De Lucia F. C., Lovas F. J., Drouin B. J., and Pearson J. C. Acetone: laboratory assignments and predictions through $620 \mathrm{GHz}$ for the vibrational-torsional ground state // Astrophys. J. Suppl. Ser. 2002. - Vol. 142, No. 1. - P. 145-151. DOI: 10.1086/341221

9. Groner P., Herbst E., De Lucia F. C., Drouin J., and Mäder $H$. Rotational spectrum of acetone, $\mathrm{CH}_{3} \mathrm{COCH}_{3}$, in the first torsional excites state // J. Mol. Struct. 2006. - Vol. 795, Is. 1-3. - P. 173-178. DOI: 10.1016/ j.molstruc.2006.02.028

10. Groner P., Medvedev I. R., De Lucia F. C., and Drouin J. Rotational spectrum of acetone, $\mathrm{CH}_{3} \mathrm{COCH}_{3}$, in the 
$v_{17}$ torsional excites state // J. Mol. Spectrosc. 2008. - Vol. 251, Is 1-2. - P. 180-184. DOI: 10.1016/ j.jms.2008.02.018

11. Groner $P$. Large-amplitude motion tunneling parameters in effective rotational Hamiltonians from rotation-internal rotation theory // J. Mol. Spectrosc. - 1992. - Vol. 156, Is. 1. - P. 164-189. DOI: 10.1016/0022-2852(92)90101-S

12. Groner $P$. Effective rotational Hamiltonian for molecules with two periodic large-amplitude motions // J. Chem. Phys. - 1997. - Vol. 107, Is. 12. - P. 4483-4498. DOI: $10.1063 / 1.474810$

13. Ilyushin V. V., and Hougen J. T. A fitting program for molecules with two equivalent methyl tops and $C_{2 v}$ pointgroup symmetry at equilibrium: Application to existing microwave, millimeter, and sub-millimeter wave measurements of acetone // J. Mol. Spectrosc. - 2013. Vol. 289. - P. 41-49. DOI: 10.1016/j.jms.2013.05.012

14. Alekseev E. A., Motiyenko R. A., and Margulès L. Millimeter- and submillimeter-wave spectrometers on the basis of direct digital frequency synthesizers // Radio Physics and Radio Astronomy. - 2012. - Vol. 3, Is. 1. - P. 75-88. DOI: $10.1615 /$ RadioPhysicsRadioAstronomy.v3.i1.100

15. Li-Hong Xu., Fisher J., Lees R. M., Shi H. Y., Hougen J. T., Pearson J. C., Drouin B. J., Blake G. A., and Braakman R. Torsion-rotation global analysis of the first three torsional states $\left(\mathrm{v}_{\mathrm{t}}=0,1,2\right)$ and terahertz database for methanol // J. Mol. Spectrosc. - 2008. - Vol. 251, Is. 1-2. - P. 305-313. DOI: $10.1016 /$ j.jms.2008.03.017

16. Groner P. Experimental two-demensional torsional potential function for the methyl internal rotors in acetone // J. Mol. Struct. - 2000. - Vol. 550-551. - P. 473-479. DOI: 10.1016/S0022-2860(00)00507-X

17. Smirnov I. A., Alekseev E. A., Ilyushin V. V., Margulès L., Motiyenko R. A., and Drouin B. J. Spectroscopy of the ground, first and second excited torsional states of acetaldehyde from 0.05 to $1.6 \mathrm{THz} / / \mathrm{J}$. Mol. Spectrosc. - 2014. - Vol. 295. - P. 44-50. DOI: 10.1016/ j.jms.2013.11.006

18. Ilyushin V. V., Endres C. P., Lewen F., Schlemmer S., and Drouin B. J. Submillimeter wave spectrum of acetic acid // J. Mol. Spectrosc. - 2013. - Vol. 290. - P. 31-41. DOI: 10.1016/j.jms.2013.06.005

19. Groner P. and Durig J. R. Analysis of torsional spectra of molecules with two internal $\mathrm{C} 3 \mathrm{v}$ rotors. II. Far infrared and low frequency Raman spectra of dimethylether isotopes // J. Chem. Phys. - 1977. - Vol. 66, Is. 5. P. 1856-1874. DOI: $10.1063 / 1.434184$

20. Groner P. Chapter 6. Internal rotation of molecules with two C3v rotors. In: Vibrational Spectra and Structure, Vol 9. Amsterdam: Elsevier, 1981. - P. 405-496.

\section{REFERENCES}

1. COMBES, F., GERIN, M., WOOTEN, A., WLODARCZAK, G., CLAUSSET, F. and ENCRENAZ, P. J., 1987. Acetone in interstellar space. Astron. Astrophys. vol. 180, no. $1-2$, pp. 13-16.

2. SNYDER, L. E., LOVAS, F. J., MEHRINGER, D. M., MIAO, N. Y., KUAN, Y.-J., HOLLIS, J. M. and JEWELL, P. R., 2002. Confirmation of interstellar acetone. Astrophys. J. vol. 578, no. 1, pp. 245-255. DOI: http:// dx.doi.org/10.1086/342273
3. FRIEDEL, D. N., SNYDER, L. E., REMIJAN, A. J. and TURNER, B. E., 2005. Detection of interstellar acetone toward the Orion-KL hot core. Astrophys. J. vol. 632, no. 1. pp. L95-L98. DOI: http://dx.doi.org/10.1086/497986

4. SWALEN, J. D. and COSTAIN, C. C., 1959. Internal rotation in molecules with two internal rotors: microwave spectrum of acetone. J. Chem. Phys. vol. 31, pp. 1562-1574. DOI: http://dx.doi.org/10.1063/1.1730653

5. NELSON, R. and PIERCE, L., 1965. Microwave spectrum, structure, and barrier to internal rotation of acetone. J. Mol. Spectrosc. vol. 18, is. 3, pp. 344-352. DOI: 10.1016/0022-2852(65)90144-X

6. VACHERAND, J. M., VAN EIJCK, B. P., BURIE J. and DEMAISON, J., 1986. The rotational spectrum of acetone: internal rotation and centrifugal distortion analysis. J. Mol. Spectrosc. vol. 118 , is. 2, pp. 355-362. DOI: 10.1016/0022-2852(86)90175-X

7. OLDAG, F. and SUTTER, D. H., 1992. The rotational zeeman effect in acetone, its g-tensor, its magnetic susceptibility anisotropies and its molecular electric quadrupole moment tensor; a high resolution microwave fourier transform study. Z. Naturforch. A. vol. 47, is. 3, pp. 527-532. DOI: 10.1515/zna-1992-0315

8. GRONER, P., ALBERT, S., HERBST, E., DE LUCIA, F. C., LOVAS, F. J., DROUIN, B. J. and PEARSON, J. C., 2002. Acetone: laboratory assignments and predictions through $620 \mathrm{GHz}$ for the vibrational-torsional ground state. Astrophys. J. Suppl. Ser. vol. 142, no. 1, pp. 145-151. DOI: $10.1086 / 341221$

9. GRONER, P., HERBST, E., DE LUCIA, F. C., DROUIN, J. and MÄDER, H., 2006. Rotational spectrum of acetone, $\mathrm{CH}_{3} \mathrm{COCH}_{3}$, in the first torsional excites state. J. Mol. Struct. vol. 795 , is 1-3, pp. 173-178. DOI: $10.1016 /$ j.molstruc.2006.02.028

10. GRONER, P., MEDVEDEV, I. R., DE LUCIA, F. C. and DROUIN, J., 2008. Rotational spectrum of acetone, $\mathrm{CH}_{3} \mathrm{COCH}_{3}$, in the $v_{17}$ torsional excites state. J. Mol. Spectrosc. vol. 251 , is. 1-2, pp. 180-184. DOI: $10.1016 /$ j.jms.2008.02.018

11. GRONER, P., 1992. Large-amplitude motion tunneling parameters in effective rotational Hamiltonians from rotation-internal rotation theory. J. Mol. Spectrosc. vol. 156, is. 1, pp. 164-189. DOI: 10.1016/0022-2852(92)90101-S

12. GRONER, P., 1997. Effective rotational Hamiltonian for molecules with two periodic large-amplitude motions. J. Chem. Phys. vol. 107, is. 12, pp. 4483-4498. DOI: $10.1063 / 1.474810$

13. ILYUSHIN, V. V. and HOUGEN, J. T., 2013. A fitting program for molecules with two equivalent methyl tops and $\mathrm{C}_{2 \mathrm{v}}$ point-group symmetry at equilibrium: Application to existing microwave, millimeter, and sub-millimeter wave measurements of acetone. J. Mol. Spectrosc. vol. 289, pp. 41-49. DOI: 10.1016/j.jms.2013.05.012

14. ALEKSEEV, E. A., MOTIYENKO, R. A. and MARGULÈS, L., 2012. Millimeter- and submillimeter-wave spectrometers on the basis of direct digital frequency synthesizers. Radio Physics and Radio Astronomy. vol. 3, is 1, pp. 75-88. DOI: $10.1615 /$ RadioPhysicsRadioAstronomy.v3.i1.100

15. LI-HONG, XU., FISHER, J., LEES, R. M., SHI, H. Y., HOUGEN, J. T., PEARSON, J. C., DROUIN, B. J., BLAKE, G. A. and BRAAKMAN, R., 2008. Torsion-rotation global analysis of the first three torsional states 
$\left(\mathrm{v}_{\mathrm{t}}=0,1,2\right)$ and terahertz database for methanol. J. Mol. Spectrosc. vol. 251 , is. $1-2$, pp. $305-313$. DOI: $10.1016 /$ j.jms.2008.03.017

16. GRONER, P., 2000. Experimental two-demensional torsional potential function for the methyl internal rotors in acetone. J. Mol. Struct. vol. 550-551, pp. 473-479. DOI: 10.1016/S0022-2860(00)00507-X

17. SMIRNOV, I. A., ALEKSEEV, E. A., ILYUSHIN, V. V., MARGULÈS, L., MOTIYENKO, R. A. and DROUIN, B. J., 2014. Spectroscopy of the ground, first and second excited torsional states of acetaldehyde from 0.05 to $1.6 \mathrm{THz}$. J. Mol. Spectrosc. vol. 295, pp. 44-50. DOI: 10.1016/j.jms.2013.11.006

18. ILYUSHIN, V. V., ENDRES, C. P., LEWEN, F., SCHLEMMER, S. and DROUIN, B. J., 2013. Submillimeter wave spectrum of acetic acid. J. Mol. Spectrosc. vol. 290, pp. 31-41. DOI: 10.1016/j.jms.2013.06.005

19. GRONER, P. and DURIG, J. R., 1977. Analysis of torsional spectra of molecules with two internal $\mathrm{C} 3 \mathrm{v}$ rotors. II. Far infrared and low frequency Raman spectra of dimethylether isotopes. J. Chem. Phys. vol. 66, is. 5, pp. 1856-1874. DOI: 10.1063/1.434184

20. GRONER, P., 1981. Chapter 6. Internal rotation of molecules with two C3v rotors. In: Vibrational Spectra and Structure, vol. 9. Amsterdam: Elsevier, pp. 405-496.

\section{Ю. А. Армеева ${ }^{1}$, В. В. Илюшин ${ }^{1}$, Е. А. Алексеев ${ }^{1,2}$, О. А. Доровская ${ }^{1}$, Л. Маргулес ${ }^{3}$, Р. А. Мотиенко ${ }^{3}$}

${ }^{1}$ Радиоастрономический институт НАН Украины, ул. Краснознаменная, 4, г. Харьков, 61002, Украина

${ }^{2}$ Харьковский национальный университет имени В. Н. Каразина, пл. Свободы, 4, г. Харьков, 61022, Украина

3 Лаборатория физики лазеров, атомов и молекул, Университет Лилль 1, F-59655 Вильнёв д’Аск, Франция

\section{СПЕКТРОСКОПИЯ ОСНОВНОГО, ПЕРВОГО И ВТОРОГО ВОЗБУЖДЕННЫХ ТОРСИОННЫХ СОСТОЯНИЙ АЦЕТОНА В МИЛЛИМЕТРОВОМ ДИАПАЗОНЕ ДЛИН ВОЛН}

Предмет и цель работы: исследование спектра первых трех торсионных состояний ацетона $\left(\mathrm{CH}_{3} \mathrm{COCH}_{3}\right)$ в диапазонах $34 \div 150$ и $480 \div 620$ ГГц.

Методы и методология работы: Новые измерения выполнены с использованием автоматизированного спектрометра миллиметрового диапазона Радиоастрономического института НАН Украины и субмиллиметрового спектрометра лаборатории ФЛАМ (Лилль, Франция). Результаты новых измерений обработаны с помощью недавно разработанной модели для молекул с двумя эквивалентными метильными группами и равновесной симметрией $\mathrm{C}_{2 \mathrm{v}}$ (программа PAM_C2v_2tops). Результаты работы: Анализ спектра молекулы ацетона выполнен с использованием как новых измерений для торсионно-вращательных переходов в миллиметровом диапазоне длин волн, относящихся к основному, первому и второму возбужденным торсионным состояниям, так и ранее опубликованных данных. Кроме того, проведены более точные измерения целого ряда ранее опубликованных линий, которые представляли некоторую проблему в предыдущем анализе с использованием той же модели.
Заключение: Результаты повторных измерений показали, что имевшиеся проблемы описания спектра были вызваны недооцененными экспериментальными погрешностями опубликованных ранее данных. Финальная модель использует 99 параметров и обеспечивает взвешенное среднеквадратичное отклонение 0.78 для набора данных, состоящего из 6233, 4868 и 4364 переходов с $J \leq 60$ и $K_{a} \leq 35$, относящихся соответственно к основному, первому и второму возбужденным торсионным состояниям молекулы ацетона.

Ключевые слова: ацетон, миллиметровый спектр, внутреннее вращение метильной группы

Ю. А. Армєєва ${ }^{1}$, В. В. Ілюшин ${ }^{1}$, С. А. Алєксеєв
О.,
О. А. Доровська
, Л. Маргулес
, Р. О. Мотієнко

${ }^{1}$ Радіоастрономічний інститут НАН України, вул. Червонопрапорна, 4, м. Харків, 61002, Україна

${ }^{2}$ Харківський національний університет імені В. Н. Каразіна,

м. Свободи, 4, м. Харків, 61022, Україна

3 Лабораторія фізики лазерів, атомів та молекул, Університет Лілль 1, F-59655 Вільньов д’Аск, Франція

СПЕКТРОСКОПІЯ ОСНОВНОГО, ПЕРШОГО ТА ДРУГОГО ЗБУДЖЕНИХ ТОРСІЙНИХ СТАНІВ АЦЕТОНУ У МІЛІМЕТРОВОМУ ДІАПАЗОНІ дОВЖИН ХВИЛЬ

Предмет і мета роботи: дослідження спектра перших трьох торсійних станів ацетону $\left(\mathrm{CH}_{3} \mathrm{COCH}_{3}\right)$ у діапазонах $34 \div 150$ та $480 \div 620$ ГГц.

Методи та методологія роботи: Нові вимірювання виконано з використанням автоматизованого спектрометра міліметрового діапазону Радіоастромічного інститута НАН України та субміліметрового спектрометра лабораторії ФЛАМ (Лілль, Франція). Результати нових вимірювань оброблено з використанням нещодавно розробленої моделі для молекул 3 двома еквівалентними метильними групами та рівноважною симетрією $\mathrm{C}_{2 \mathrm{v}}$ (програма PAM_C2v_2tops).

Результати роботи: Аналіз спектра молекули ацетону виконано з використанням як нових вимірювань для торсійнообертальних переходів у міліметровому діапазоні довжин хвиль, що належать до основного, першого та другого збуджених торсійних станів, так і раніше опублікованих даних. До того ж, виконано точніші вимірювання низки раніше опублікованих ліній, котрі становили деяку проблему в попередньому аналізі з використанням тієї ж моделі.

Висновок: Результати повторних вимірювань показали, що наявні проблеми з описом спектра було спричинено недооцінкою експериментальних похибок раніше опублікованих даних. Фінальна модель використовує 99 параметрів та забезпечує зважене середньоквадратичне відхилення 0.78 для набору даних, що містить 6233,4868 та 4364 переходів з $J \leq 60$ та $K_{a} \leq 35$, які належать відповідно до основного, першого та другого збуджених торсійних станів молекули ацетону.

Ключові слова: ацетон, міліметровий спектр, внутрішнє обертання метильної групи 